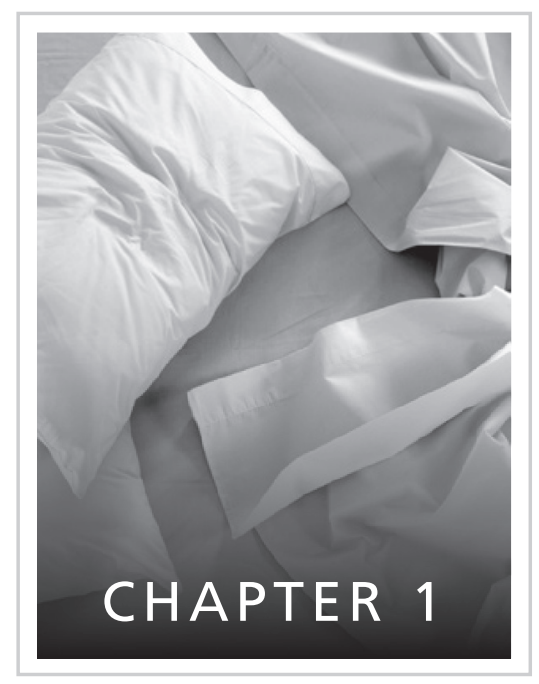

\title{
Will This Book Help You?
}

Y ou may be at your wits' end, thinking that you have tried everything to sleep better, and nothing has really worked. Or you may be optimistic, believing that there are things you have not yet tried that may improve your sleep. Or you may be somewhere in between. Wherever you are, it's perfectly fine to be there. The strategies in the next chapters work even, or especially, if you are a bit skeptical to begin with.

If you are "at your wits' end"- -hang in there. Just by opening this book, you are demonstrating hope and a belief that things can change for the better. All that's needed is having your mind open to the possibility that your sleep will improve. However, you don't need to be gung ho about it. In fact, it is better if you are relatively relaxed and take somewhat of an observer stance. If you are thinking, "That's not me! I can't relax," take a deep breath and consider the notion that your body and mind do have the capacity for good sleep, and that by using some specific techniques, you will encounter evidence of this.

If you are "optimistic" — then, good for you! Keep that style of thinking. You can use your energy and enthusiasm to design your own sleep program. You need to have a bit of patience, though, as this is not an overnight fix for your sleep. Rest assured that you will see positive results as you consistently apply the techniques.

If you are "somewhere in between"—not completely discouraged and not overly enthusiastic - you may be in the best position to benefit from sleep strategies. You have the energy to try some things, but in a somewhat experimental way, with an 
ability to work with "what is" and an openness to discovering how sleep therapy works and to identifying the techniques that work best for you.

\section{THE TECHNIQUES WORK}

Years of research have revealed that certain techniques reliably lead to improved quality and quantity of sleep and increased satisfaction with sleep. Together these techniques can be called "cognitive behavioral therapy for insomnia," which I will now call CBT-I. In the mid-1990s two important reports were published on these techniques. These reports were based on meta-analyses. In a meta-analysis, the research data from many studies of a given treatment are carefully combined to provide the overall story on the treatment's usefulness. In these particular meta-analyses, the authors reviewed studies that had compared CBT-I techniques with no treatment or with a placebo treatment. These two meta-analyses were carried out independently, one in North America and one in Australia. It was exciting when the results came out (they were published within months of each other). The reports reached similar conclusions: CBT-I produced significant improvements in sleep for people with insomnia. With CBT-I, people fell asleep faster, were less likely to wake up during the night, and had a longer nightly sleep duration. More exciting was the evidence that sleep improvements were well maintained, and sometimes enhanced, 6-8 months after people had learned the techniques.

Since then, many more reviews and meta-analyses have confirmed that there is sound scientific evidence for the effectiveness of CBT-I techniques. The recent body of research tells the same story, that people with persistent insomnia who use the techniques reduce their time to fall asleep, their awakenings during the night, and their time awake in bed. They get more solid, satisfactory sleep. Because of its proven effectiveness, medical guidelines in North America and Europe now recommend CBT-I as the first-line treatment for persistent insomnia, above any sleep medication. That means it is the first-choice, standard treatment. We also now have more evidence that CBT-I's benefits are not limited to research facilities: it works very well when provided by nurses, psychologists, or other health professionals in real-life clinical settings, like family doctors' offices. Furthermore, we now have confirmation that people who do self-help CBT-I, by following a program like the one in this book, improve their sleep significantly. They fall asleep faster, sleep more solidly during the night, and experience enhanced sleep quality. People tend to like these techniques, preferring them to sleep medication. In sum, the evidence is consistently positive for the effectiveness of CBT-I. It is high time that these techniques were widely accessible to people who need them.

\section{IMPROVEMENTS ARE FASTER THAN YOU MIGHT THINK}

Many people who need these techniques do not know about them. Family physicians are the health professionals who most often hear about sleep problems. But family physicians are very busy people; they usually do not have the time to learn or to support patients with sleep treatments other than drugs. They sometimes 
prescribe sleeping pills, also called hypnotic medication. Hypnotic medication is good for the short term only, up to 4 weeks. This book will show you how to deal with and overcome your insomnia if you have insomnia that has lasted longer than 4 weeks. The approaches I will introduce you to don't work overnight. However, it does not take long to see improvements in your sleep with CBT-I. Most people in the clinic start sleeping better within 2 weeks of trying the techniques. After discovering the strategies that work best for their situation, they simply fine-tune them. The vast majority of people who use these techniques no longer have insomnia after 5 weeks. Perhaps 5 weeks will feel like a small investment of your time given the benefits of good sleep for years ahead? You are about to find out.

\section{YES, THERE ARE SOME POSSIBLE SIDE EFFECTS}

Although CBT-I is the best and most durable treatment we have for insomnia, it can have side effects. When people carry out "sleep restriction therapy," which is a component of CBT-I, they sometimes experience excessive sleepiness, reduced vigilance, and slowed reaction time. These effects are especially likely to occur at the beginning of the program, and they disappear when sleep is no longer restricted. If the notion of sleep restriction sounds awful to you, it may help to know that this is not total sleep deprivation by any means. The technique is designed to limit the degree of sleep restriction to what has already been occurring with your insomnia. Sleep restriction therapy is also meant to be used for a short time (usually 2-4 weeks), and should not be continued if your sleep has not improved substantially in that time. When we get to the chapters that involve sleep restriction therapy, I will be reminding you to avoid driving or other dangerous activities when you are sleepy.

\section{GOOD SLEEP CAN BE MAINTAINED}

There is evidence that by using CBT-I approaches, people not only maintain their good sleep over time-the current research has followed people up to 2 years-but they often see their sleep continuing to improve! My experience is that once people understand their sleep problem and identify the combination of simple strategies that works for them, they remember and use these strategies when faced with occasional sleep difficulty months and years later. By doing so, they prevent relapses into full-blown insomnia.

Let me now address a few concerns that are quite common when people first consider CBT-I.

\section{YOU ARE NOT A HOPELESS CASE-THERE ARE NO HOPELESS CASES}

People sometimes feel that their particular case of insomnia is hopeless. This idea is expressed by people who have had poor sleep for many years despite having tried "everything." First, let me say that even if you have had long-term insomnia, these techniques still work. The research results are somewhat unclear about 
whether or not you will take a bit longer to improve your sleep, but is very clear that you will still benefit. In some of my research with insomnia expert Charles Morin, people arriving at his clinic had been experiencing sleep difficulty for an average of 11 years! It is not uncommon for people to arrive at our Kingston clinic with insomnia that began decades ago, for example, when they were studying for exams as a young adult or when they had a baby who is now grown-up. People whose insomnia had persisted for many years have used these techniques very successfully.

Many people who come to the clinic have already tried various strategies to obtain better sleep. Usually they have tried very reasonable approaches like avoiding caffeine, having a warm bath in the evening, using relaxation apps, listening to music, doing yoga, getting cardiovascular exercise to tire themselves out, stretching, avoiding stimulating activities in the evening, and turning off their electronic devices an hour before bed. They also have tried strategies while in bed trying to sleep, like counting sheep, deep breathing, listening to music, turning on a fan for white noise, and so on. One person was convinced that slowly stretching the whole body just before sleep, like a cat, promotes sleep. However, I have noticed that cats tend to stretch after rather than before sleeping. (To be like a cat we would need to make three turns before settling down and then spend a few moments licking our feet!)

Although such strategies may be helpful, they do not cure chronic insomnia. They may be helpful for people when they are feeling stressed and have situational insomnia, but research has shown that they are not enough to reverse persistent insomnia. Many of the strategies of CBT-I are not yet well known but that will soon change. The effective strategies are the ones that I will introduce you to in the chapters ahead.

\section{YOU ARE NOT TOO OLD TO START}

This is not just an approach for young people. There is abundant evidence that middle-aged and older adults with insomnia benefit from CBT-I and that the beneficial effects endure. As we age we are more and more likely to have difficulties sleeping through the night. CBT-I improves sleep quality and reduces time awake during the night. We have seen people in their 80 s successfully improve their sleep with CBT-I. It is safer than using sleeping medications. Sleeping pills can cause side effects that are particularly worrisome for older people, including memory problems, unsteadiness, falls leading to broken bones and other injuries, impaired driving, and addiction to the medication.

\section{THERE PROBABLY ISN'T A CHEMICAL IMBALANCE}

The body, including the brain, shows signs of "hyperarousal" with insomnia. For example, there may be increased levels of the hormone cortisol, increased heart rate, and increased strength of high-frequency brain waves. However, these are correlations rather than cause-and-effect relationships. The cases where a chemical imbalance in the brain produces persistent insomnia are rare. Although insomnia 
can accompany illness, there are not many conditions that directly and specifically cause insomnia through a chemical imbalance. The closest thing might be a disease called "fatal familial insomnia," which causes an inability to sleep. This is a genetically inherited prion (infectious protein) disease. The incidence of this disease is so rare that it is not documented; it is definitely less than 1 in 100,000 and probably less than 1 in 1 million. If you have this disease, you would have already turned yourself over to physicians for help. So, if you are reading this book, chances are that your sleep difficulty is not caused by a chemical imbalance. It is more likely related to factors that I outline in the chapters ahead and will therefore respond to CBT-I.

\section{YOU'VE BEEN TAKING SLEEP MEDICATION. THAT'S OKAY}

Research shows that the same CBT-I techniques work regardless of whether you are taking sleeping medication or not. Based on what we see in the clinic, people who take prescribed sleeping pills occasionally, or on fewer than half the nights in a week, reduce their use of sleep medication gradually over a few weeks as they use CBT-I and discover that they can sleep without the medication. It is a bit different for people who have been taking sleeping medication every night for several months or years. If you are one of these people and are reading this book, it means that you are not entirely happy with your sleep and therefore your nightly use of sleeping pills is no longer helping. In this case, I encourage you to consider slowly withdrawing from the medication in consultation with your family physician and/or pharmacist. Longterm use of sleep medication can lead to tolerance (needing more of the drug to get the same effect) and dependence (not being able to sleep without the medication). If you are ready to discontinue sleep medication that you've used nightly for several months, it is of utmost importance that you do so in a gradual and planned way with medical guidance. This means over weeks or months. Otherwise there will be uncomfortable withdrawal symptoms including much-worsened insomnia. You can do CBT-I as you follow a medication tapering schedule designed by your pharmacist or physician. You will thereby be immediately learning sleep techniques that will replace the need for medication.

\section{YOU HAVE OTHER HEALTH OR MOOD ISSUES. THAT'S OKAY}

If you are a normal human being, you have other problems besides trouble sleeping. Research shows that CBT-I is useful even if you have some mild to moderate symptoms of anxiety or depression or some long-lasting medical problems like chronic pain or cancer. In fact, there is some evidence that people with coexisting health or mood conditions show greater improvements in their sleep with these techniques than do people who have "just" insomnia! Chapters 22 and 23 will describe how to tailor your program to your situation.

All that being said, there are some specific circumstances in which this Sleep Therapy program may not be enough to help your sleep. 


\section{CIRCUMSTANCES WHEN THIS BOOK MAY NOT BE ENOUGH}

If you are severely depressed and have not received any treatment yet, you need to visit your family physician and get advice on the best available treatment. By severely depressed, I mean feeling so low that you are having difficulty getting out of bed every day, not functioning as usual, not able to go to work, or feeling hopeless or suicidal. You should know that support is available and that there are effective treatments for depression, some involve medications, and some involve psychotherapy (e.g., cognitive behavioral therapy or interpersonal therapy). Your family physician is the first person to talk with about the options in your community.

Another situation in which you may need special support and assistance is bereavement. If someone close to you has recently died, then you are likely to be grieving and this needs time and special attention; it is not the best time to do this program.

If you have experienced or witnessed an extremely traumatic event that has left you feeling vulnerable and jumpy, distressed or detached, and you have intense recollections, flashbacks, or nightmares related to the trauma, then you need to receive support from someone who understands trauma. That being said, recent research indicates that you can benefit from CBT-I, so it is really up to you whether you do CBT-I now or later. In any case, I recommend that you also have support from a counselor or therapist with expertise in trauma.

If you need alcohol or other non-prescribed substances or drugs in order to get through the day, or if your use of these substances is interfering with your day-today functioning, then the CBT-I techniques will probably not be appropriate for you. That is because most, if not all, recreational drugs affect sleep directly and compromise people's ability to follow this program. If you are a smoker, the CBT-I techniques will probably help your sleep. However, I recommend for your own health and well-being that you consider a smoking cessation program. There is support in your community; start with your family physician or your local health unit. I would not recommend that you try to stop smoking or any other psychoactive substance at the same time as taking this sleep program. Changing one behavior at a time is much more manageable.

If you are troubled by obsessions (have difficulty letting go of certain thoughts, impulses, or images-ones that go around and around in your head or that pop into your mind when you don't want them there) or by compulsions (feeling compelled to do certain things over and over before you feel comfortable, such as checking, counting, pairing things up in your mind, washing your hands many more times than needed), you should know that there is help for these obsessive-compulsive symptoms. It would be wise to receive help for them first, before following this CBT-I program. Your family physician would be the place to start if you have not already received help.

If there is a medical condition that directly causes the insomnia, for example, untreated hyperthyroidism or acute, unrelenting pain due to a bone fracture or 
injury, then the insomnia will likely continue until the condition is treated or controlled. Also, certain medications such as corticosteroid medication can cause sleeplessness. If your insomnia started around the time of a new medication and it persists only with the medication, then discuss this with the physician who prescribed that medication, your family physician, or your pharmacist. They may suggest alterations to the dose, timing, or other factors that will allow you to return to good sleep.

Sometimes other sleep disorders masquerade as insomnia. These other sleep disorders have treatments of their own. Periodic limb movement disorder is a problem in which the person's legs twitch or make small jerks repetitively during sleep. This can disrupt sleep even if the person is unaware of the movements, and can cause an unsatisfying, light sleep. Many people who have restless legs syndrome-a restless feeling in the legs that urges them to walk or move around-also have periodic limb movement disorder. Sleep apnea is another fairly common sleep disorder, which involves loud snoring and a cessation of breathing for repeated periods of at least 10 seconds. This sleep disorder, especially when severe, can disrupt sleep, cause daytime sleepiness, and affect how you function at work, school, or home. Sleep apnea needs to be treated so you can feel better. Treatment starts with visiting your family physician. They may refer you to a clinical sleep laboratory. There are effective treatments for these sleep disorders so it makes good sense to receive help for them. You can always come back to this CBT-I program if you still have insomnia after you have received help for the other sleep disorders.

By the way, there is NO NEED to go to a sleep laboratory if insomnia is your only sleep problem; that is, if you and your physician do not suspect another type of sleep disorder. You will probably not sleep in the lab-a finding that will confirm what you already know!

There is another sleep-wake disorder called delayed sleep phase syndrome. This occurs when people's internal 24-hour rhythm of sleep and wakefulness is delayed compared to what they wish it were. These are people who have always been "night owls" and had trouble getting up in the morning. Young adults are more likely than middle- and older-aged adults to experience this. If they had their choice, they would have a late bedtime (e.g., 2:00 a.m.-6:00 a.m.) and a late wake time (e.g., 10:00 a.m.2:00 p.m.). When they can sleep at their preferred times, their sleep is normal. However, when they try to conform to usual school or work hours, they run into problems. They go to bed earlier than is natural for them, they take a long time to fall asleep, and they have great difficulty getting out of bed in the morning, even with several alarms. If this sounds like you, you will be more likely to benefit from a technique designed to advance your sleep rhythms than from CBT-I. A professional at a sleep clinic with expertise in the treatment of circadian rhythm disorders will be able to help you. This usually involves carefully scheduled exposure to bright light.

If you are under a lot of pressure right now due to continuing stress at work or at home, or if there is an ongoing conflict, you may want to seek support with these specific issues from a good counselor. If these issues are not resolved or at least 
stable, they may overwhelm your body's ability to benefit from CBT-I just now. If you travel frequently to different time zones, then some of the most powerful strategies will not be easy to carry out, so it is best to start this program when you are based at home.

Insomnia is very common among shift workers. Most shift schedules place a demand on your sleep-wake system. Some of these involve too much variation in your sleep timing for the techniques in this book to solve your sleep problem. This is true if you are currently working rotating shifts that require you to have very different bedtimes and rise times (for example, having to change your bedtime by 4 hours or more) within a 2-week period, or if you work permanent nights and you then revert to being awake during the day on your days off. These types of shift schedules are hard on the body and the mind, so look after yourself well. It is best to get help from an organization or circadian rhythm specialist with shift-work expertise. From them, you can learn strategies for sleeping as well as possible, and for maintaining other aspects of good health such as nutrition, exercise, recreation, connections with family and your community, when you have shifting work and sleep schedules. For other types of shift work where there are no overnight shifts (for example, you move between day and evening shifts) and you can have a fairly stable bedtime and rise time, then the techniques in this book should be helpful for you.

Finally, if you have too hectic a schedule that does not allow you time to focus on the program for a few minutes each day, then you may want to delay starting this program to a time when you can maximize your success with the strategies.

If all the issues in this section are either not relevant to you, or you have dealt with them, then it is time to move ahead into your insomnia treatment program! Let's start with some information about insomnia. 